\title{
Comparison of Kidney Transplant Results in Kermanshah and Iran: A Systematic Review and Meta-Analysis Study
}

\author{
Mansour Rezaei $^{(\mathbb{1})}$, Maryam Viesmoradi*2 ${ }^{\mathbb{1}}$, Roya Safari Faramani ${ }^{3}$
}

1. Professor, Department of Biostatistics, Social Development and Health Promotion Research Center, Kermanshah University of Medical Sciences, Kermanshah, Iran

2. MSc of Biostatistics, School of Public Health, Kermanshah University of Medical Sciences, Kermanshah, Iran

3. Assistant Professor, Department of Epidemiology, School of Public Health, Kermanshah University of Medical Sciences, Kermanshah, Iran

\section{Article Information}

\begin{tabular}{lr}
\hline \multicolumn{2}{c}{ Article History } \\
Received: & $2020 / 04 / 12$ \\
Accepted: & $2020 / 05 / 14$ \\
Available Online: & $2020 / 06 / 26$
\end{tabular}

JUR 2019; 3(2):57-67

DOI: $10.30699 /$ jru.3.2.57

Use your device to scan

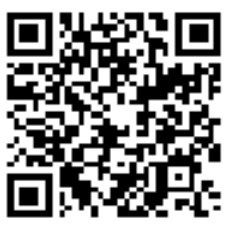

Corresponding Author

Maryam Viesmoradi MSc of Biostatistics, School of Public Health, Kermanshah University of Medical Sciences, Kermanshah, Iran Email:

\section{Abstract}

Background \& Objective: One of the most effective treatments in the case of curing the patients with advanced renal failure is kidney transplantation. Decades have passed since kidney transplants have begun in Iran and Kermanshah. The purpose of this study was to evaluate the status of Kermanshah province in comparison with other provinces and the whole country in the field of kidney transplantation.

Methods: In this systematic review and meta-analysis study, survival rate of patients after the transplantation and the transplanted kidney survival were investigated based on Iranian valid sources of information such as SID, Magiran, Irandoc, IranMedex and internationally validated databases such as PubMed, ISI and Science Direct from 2000 to 2019 surveyed. Keywords of kidney transplantation, survival rate, transplant survival rate, Kermanshah, Iran, and a combination of them were used and analyzed by Random Effects Model.

Results: In 33 studies, 21113 samples were evaluated. Survival rate of 1,3 and 5 years in patients undergoing renal transplantation in Kermanshah province during the period of 2000-2019 is lower than in other provinces. Also, in Kermanshah province, 1, 3, 5 and 10 years survival rate of all kidney transplants was lower than other provinces.

Conclusions: In Kermanshah, Survival rate of patients and transplant survival in patients is lower than other regions of Iran. Therefore, it is necessary to pay attention to effective factors in increasing survival of transplant recipients and transplant kidneys as well as increasing renal transplantation studies.

Keywords: Kidney transplant, Iran, Kermanshah, Systematic review, Meta-analysis, Survival rate, Transplant survival

How to cite this article:

Rezaei M, veismoradi M, safari R. Comparison of Kidney Transplant Results in Kermanshah and Iran: A Systematic Review and Meta-Analysis Study. J Res Urol. 2019; 3 (2) 
مقايسأ نتايج يِيوند كليه در كرمانشاه و ايران: مطالعُ مرور سيستماتيك و متآناليز

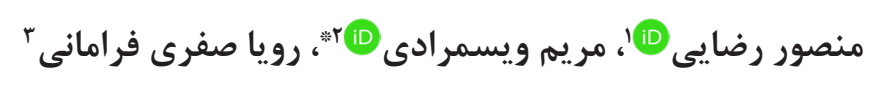

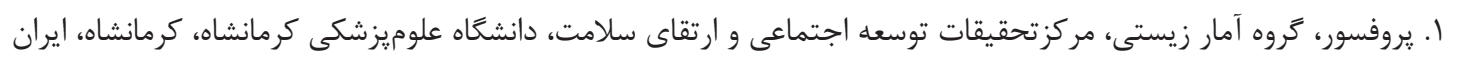

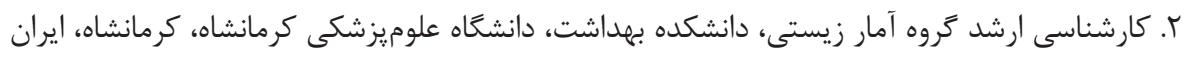

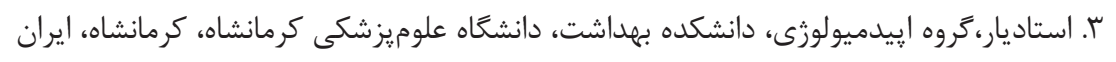

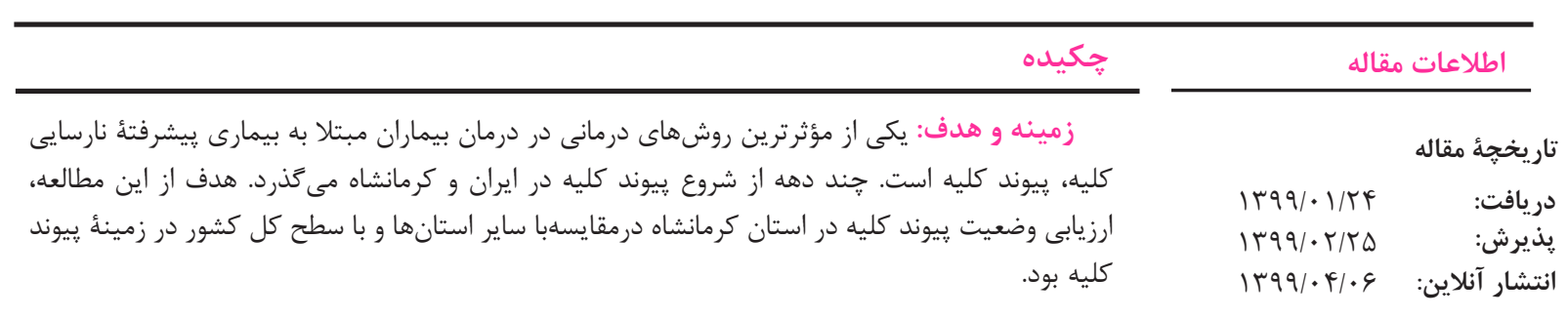

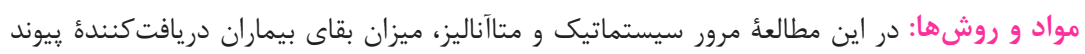

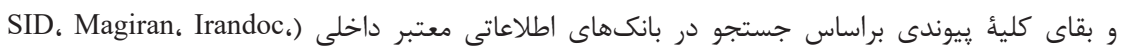
(IranMedex JUR 2019; 3(2):57-67

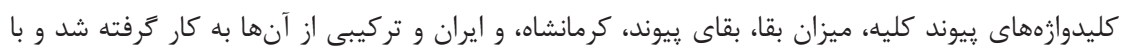
مدل اثرات تصادفى تحليل شدند.

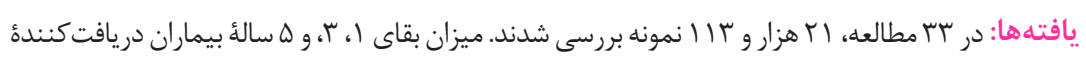

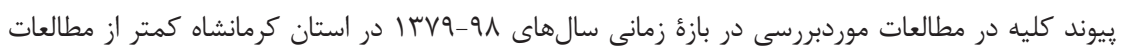

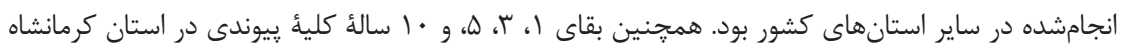
كمتر از ساير استانها بود.

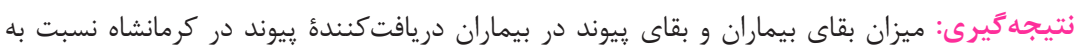

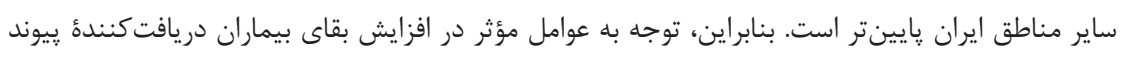

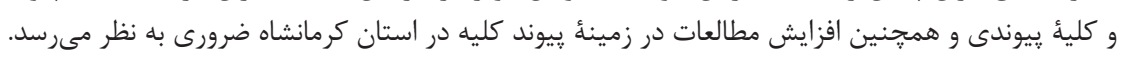
وارههاى كليدى: يِيوند كليه، ايران، كرمانشاه، مرور سيستماتيك، متآناليز، ميزان بقا، بقاى ييوند براى دانلود اين مقاله،

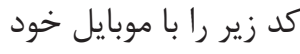

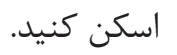

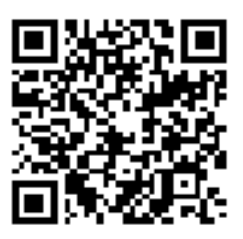

نويسنده مسئول: مريم ويسمرادى، كارشناسى ارشد كروه آمار زيستى، دانشكده بهداشت، دانشكاه علوميزشكى كرمانشاه، كرمانشاه، ايران 
مقالههاى بيشتر مرور شد. براى انتخاب مستندات، ابتدا

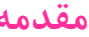

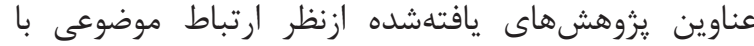

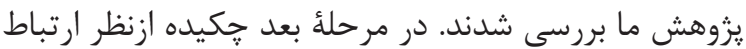

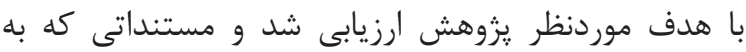

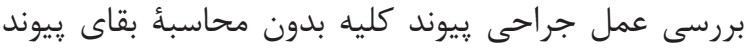

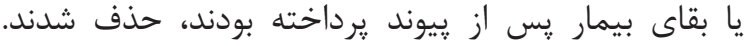

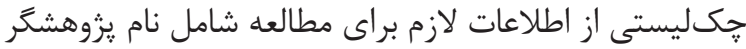

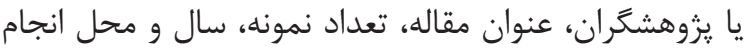

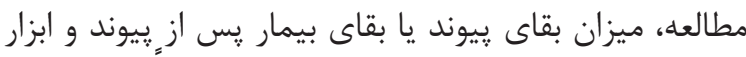

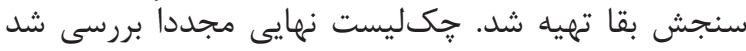

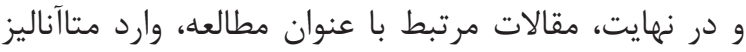

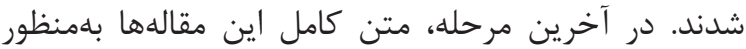

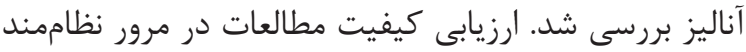

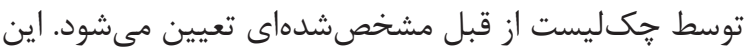

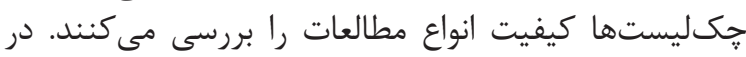

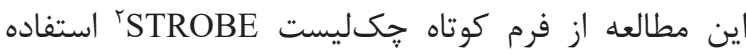

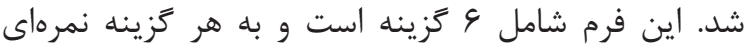

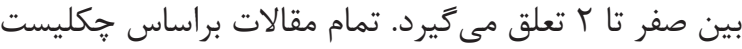

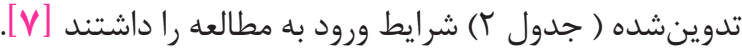

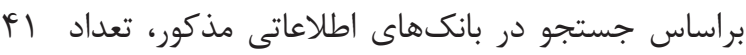

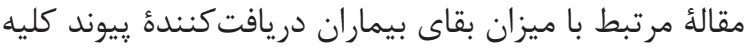

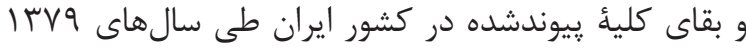

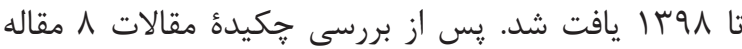

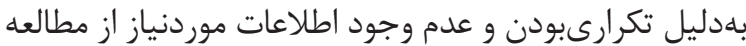

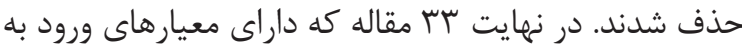

مطالعه بودند وارد تحليل متآناليز شدند (شكل (1) ).

ميزان بقاى 1 تا • اساله در مطالعات به صورت درات احتمال

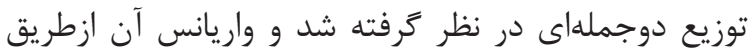

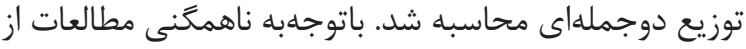

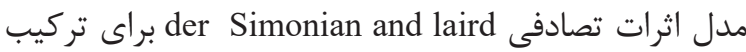

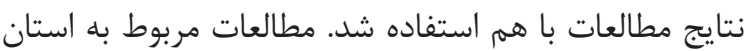

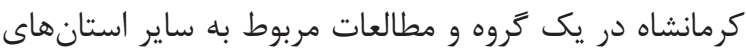

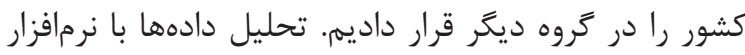

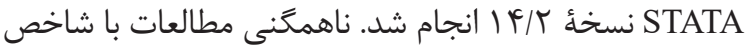

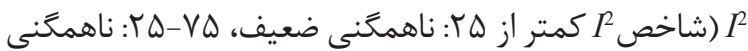

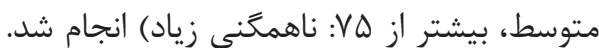

مافتنها

كل افراد شركت كننده در مطالعات بررسىشده اين

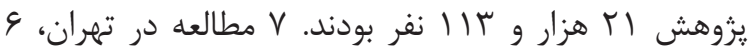

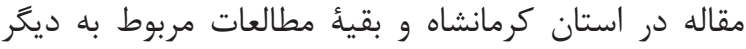

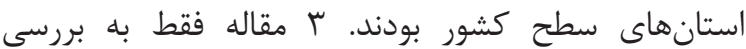

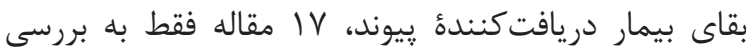

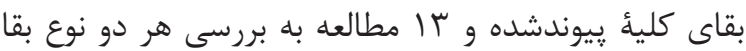

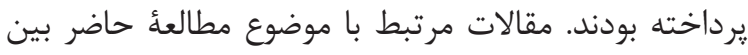

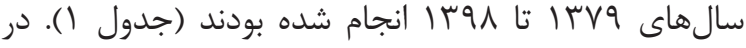
برخى مطالعات موردبررسى در جدول شماره ال، جمان انديت 2. The strengthening the reporting of observational studies in epidemiology
•ا درصد از جمعيت جهان دركير بيمارى مزمن كليه

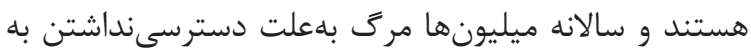

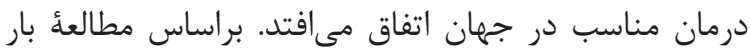

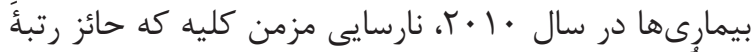

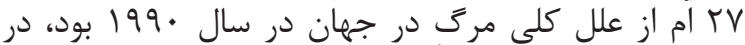

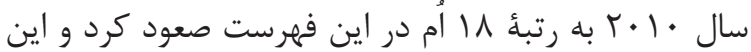

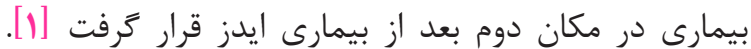

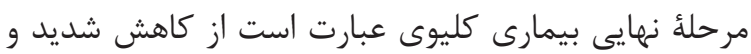

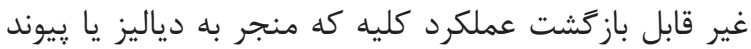

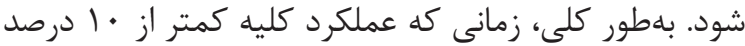

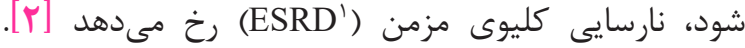

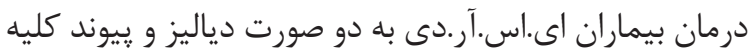

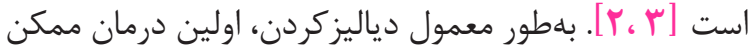

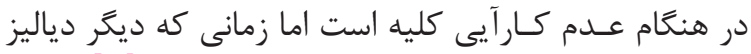

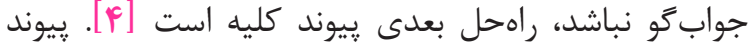

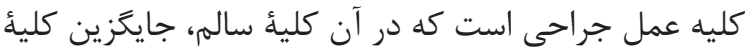

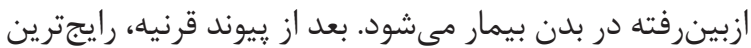

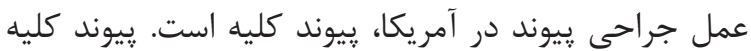

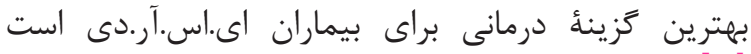

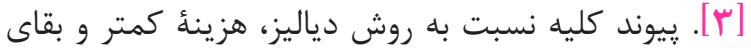

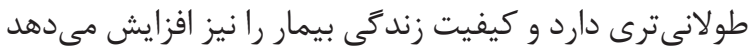

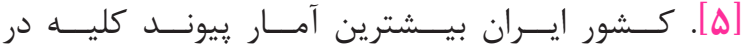

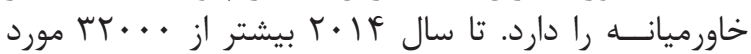

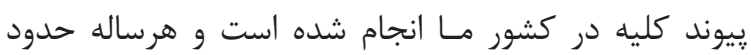

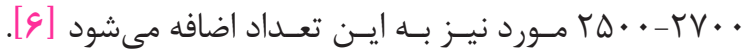

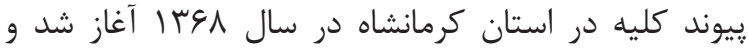

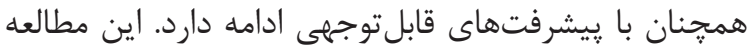

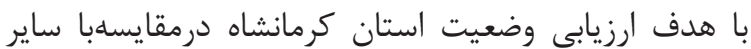

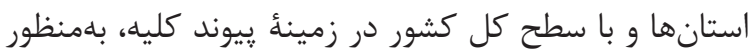

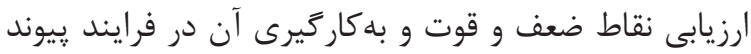
در كرمانشاه انجام شده است.

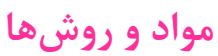

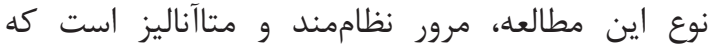
شامل جند بخش ميىشود: تعيين دقيق مسئله موردتحقيقيق،

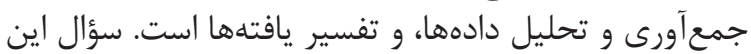

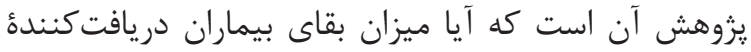

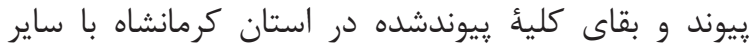

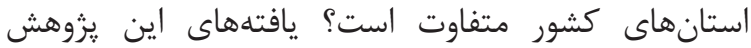

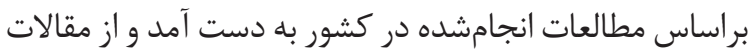

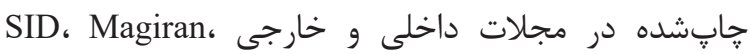
Irandoc، IranMedex،Google Scholar ،PubMed ،ISI ، ScienceDirect مقالهها بلطور عمده با استفاده از جستجوى سيستماتيك

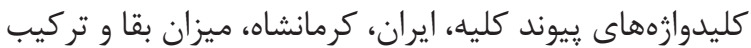

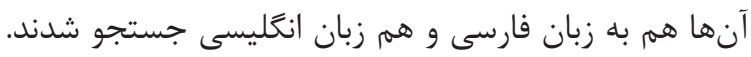
همجنين فهرست منابع مقالههاى شناسايىشده بران آنايسى يافتن 1. End Stage renal disease 


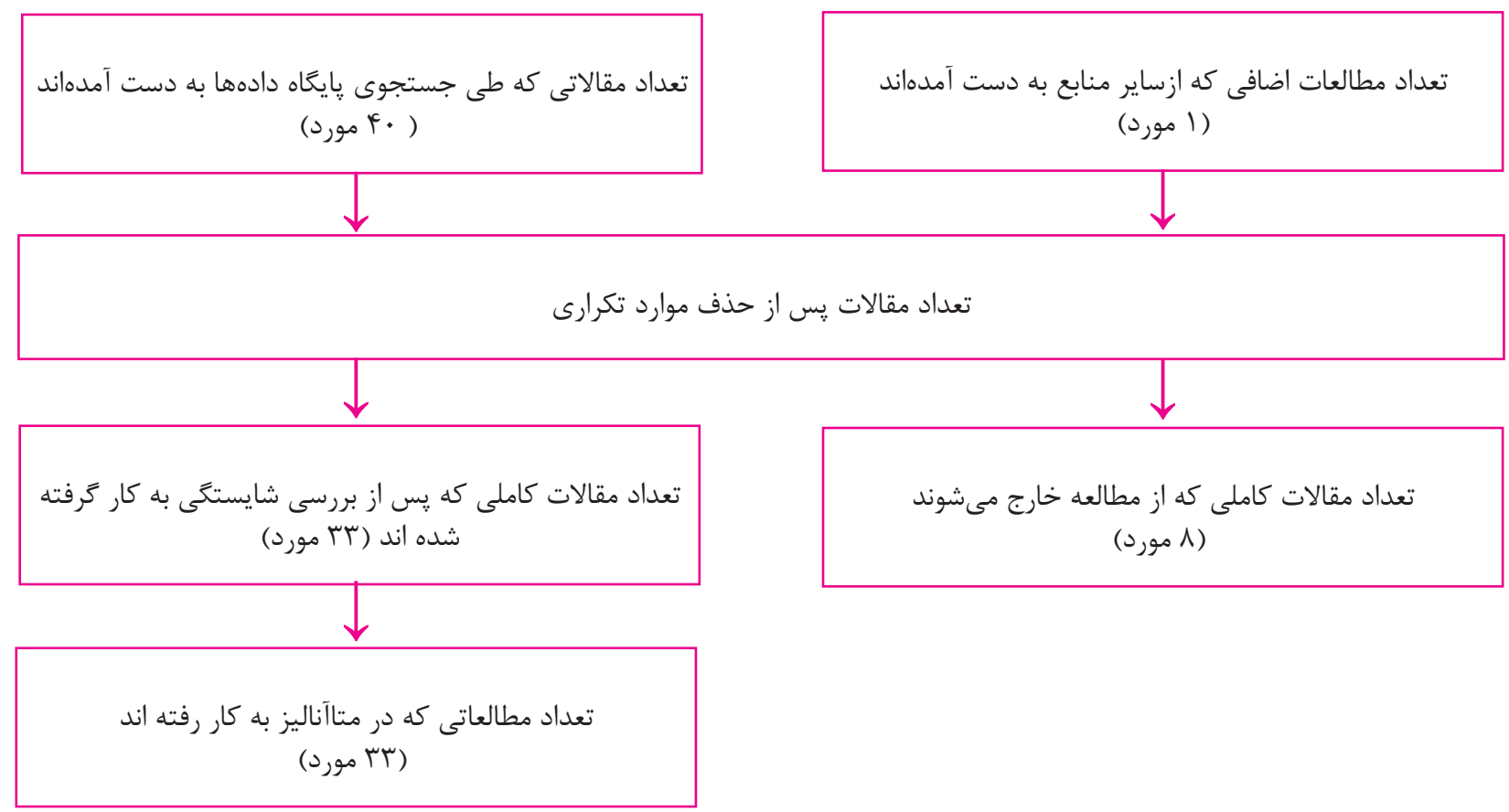

شكل ا. نمودار انتخاب مقالات

$$
\text { با ساير استانهاى كشور كمتر است (شكل ؟ ). }
$$

نمودارهاى مربوط به ميزان بقاى سه، ينج و ده سالأ بيماران

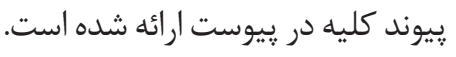

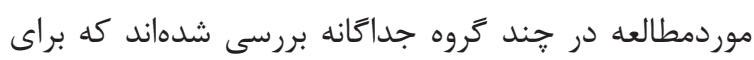

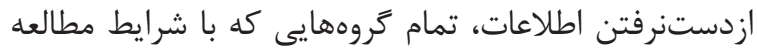

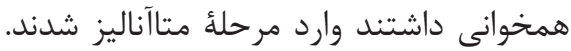
ميزان بقاى يكساله بيماران در استان كرمانشاه در مقايسه

جدول ا. مشخصات كلى مقالات موردبررسى كه شامل معيار هاى ورود به مطالعه بودند

\begin{tabular}{|c|c|c|c|c|c|c|c|c|c|c|c|}
\hline \multicolumn{2}{|c|}{ بقاى دهساله } & \multicolumn{2}{|c|}{ بقاى ينجساله } & \multicolumn{2}{|c|}{ بقاى سهساله } & \multicolumn{2}{|c|}{ بقاى يكساله } & \multirow{2}{*}{ نمونه } & \multirow{2}{*}{ سال انتشار } & \multirow{2}{*}{ نويسندة اول } & \multirow[b]{2}{*}{ مكان مطالعه } \\
\hline \multirow[t]{4}{*}{ بيوند } & بيمار & بِيوند & بيمار & بيوند & بيمار & بيوند & بيمار & & & & \\
\hline & & & & & & .199 & & 119 & Irvq & شريفى & همدان [A] \\
\hline & & & & $\cdot|\Lambda|$ & .190 &.$/ 91$ & .199 & $1 \cdot r$. & Irی. & يورشانى & [9] [9هران \\
\hline & & & & & & $\cdot 1199$ &.$/ 9 F$ & 191 & Irی. & شهبازيان & اهواز [• [1] \\
\hline.$/ 49$ & $\cdot / V r$ & .199 & • & & & - IAV & $.19 T$ & IFr. & $|r \wedge|$ & قدس & ايران [11] \\
\hline$\cdot 19$. & $\cdot / V T$ & & &.$/ 9 T$ & $.19 \mathrm{~V}$ &.$/ 9 \mathrm{~V}$ & 1 & \multirow{2}{*}{ VIr } & \multirow{2}{*}{ IrNT } & \multirow{2}{*}{ رضيى } & \multirow{2}{*}{ كرمانشاه [ I I] } \\
\hline \multirow[t]{6}{*}{ (r } &.$|9|$ & & & $\cdot / V V$ & - IAT & $\cdot \mid \Lambda \Delta$ & $\cdot 119$ & & & & \\
\hline & & $\cdot / V F$ & • & & &.$/ 9$ & $.19 T$ & \multirow{2}{*}{$|V| \overline{\mid}$} & \multirow{2}{*}{ IrNF } & \multirow{2}{*}{ عبدى ع } & \multirow{2}{*}{ تهران [זו] } \\
\hline & & $\cdot 190$ & - /AV & & & $\cdot / \Lambda \Delta$ & . 194 & & & & \\
\hline & & & & &.$/ 91$ & & $.19 f$ & $r \cdot V$ & IrAF & نجفى & تهران [If] \\
\hline & & & & & & $\cdot / \Lambda \Delta$ & .191 & \multirow{2}{*}{ rgf } & \multirow{2}{*}{ ITAV } & \multirow{2}{*}{ رضايى } & \multirow{2}{*}{ كرمانشاه [ه1] } \\
\hline & & & & & & .190 & 1 & & & & \\
\hline
\end{tabular}




\begin{tabular}{|c|c|c|c|c|c|c|c|c|c|c|c|}
\hline \multicolumn{2}{|c|}{ بقاى دهساله } & \multicolumn{2}{|c|}{ بقاى هنجساله } & \multicolumn{2}{|c|}{ بقاى سهساله } & \multicolumn{2}{|c|}{ بقاى يكساله } & \multirow[b]{2}{*}{ نمونه } & \multirow[b]{2}{*}{ سال انتشار } & \multirow[b]{2}{*}{ نويسندهُ اول } & \multirow[b]{2}{*}{ مكان مطالعه } \\
\hline بيوند & بيمار & بيوند & بيمار & بيوند & بيمار & بيوند & بيمار & & & & \\
\hline & & $\cdot / v 9$ & & •/AT & & .194 & & $r$ r. & IrAv & اتوكش & تهران [ع] \\
\hline & & & & & & .194 & $.19 V$ & $1 \Delta \Delta$ & IrAv & سليمانى & كاشان [IV] \\
\hline & & $\cdot / 9$ & & . 194 & & .199 & & MIS & $1 \mathrm{H} \wedge \Lambda$ & اشرفى & اصفهان [1/] \\
\hline & & $\cdot|4|$ & & $\cdot \mid \Delta r$ & & $\cdot / V F^{c}$ & & $\Delta \cdot$ & $1 \% \wedge 9$ & طرماسبى & اروميه [19] \\
\hline$\cdot 119$ & & .194 & & .199 & & .191 & & NFr & $1 \% \wedge 9$ & حسن زاده & شيراز [·r] \\
\hline \multirow[t]{7}{*}{$\cdot / \wedge \Delta$} & & $\cdot \mid \Lambda \Lambda$ & & .194 & &.$/ 99$ & & Irog & $1 \% 19$ & الماسى حشيانى & شيراز [إr] \\
\hline & & $.19 T$ & & & & & & $1 T \Delta \varphi$ & $11 \%$. & \multirow{3}{*}{ الماسى حشيانى } & \multirow{3}{*}{ شيراز [rr] } \\
\hline & & $.19 T$ & & & & & & Iros & $1 r q$. & & \\
\hline & & - IAT & & & & & & ITDS & $1 \% q$. & & \\
\hline & & & & $.19 V$ & . 194 & .191 & .199 & $r \cdot$. & $1 \% q$. & اكبرزاده & بابل [rr] \\
\hline & & - int & $\cdot 119$ & & & $\cdot 119$ &.$/ 9 V$ & ror & 149. & قانعى & تهران [Yr] \\
\hline & & $\cdot / V r$ & & $\cdot / V V$ & & $\cdot / \wedge \Delta$ & & $V \Delta G$ & Irar & هاشميان & كرمانشاه [ra] \\
\hline \multirow[t]{4}{*}{ - IAF } & & .191 & & & & .199 & & ITF. & IrqT & جوانروح گيوى & مشهد [عr] \\
\hline & & $\cdot / V \Delta$ & & $\cdot / \Lambda$ & & $\cdot / A V$ & & $V \Delta G$ & irgr & هاشميان & كرمانشاه [rV] \\
\hline & & $\cdot|\Lambda|$ & .194 & & &.$/ 9 T$ & .194 & gq & Irqf & جلال زاده & تهران [rی] \\
\hline & $\cdot \mid \Lambda F$ & & $\cdot 191$ & & & & qV/r. & iva & Irqf & فردمال & همدان [ra] \\
\hline$\cdot / V V$ & $\cdot|\Lambda|$ & $\cdot \mid A Y$ & $\cdot 1199$ & $\cdot \mid \Lambda \Lambda$ & $.19 T$ & .194 &.$/ 94$ & $\mid r G \Lambda$ & Irqf & قليجى قوجق & اروميه [•r] \\
\hline . 194 & & $\cdot 194$ & & $\cdot / 9 V$ & &.$/ 99$ & & rto & Irqf & شهبازى & اراى [וr] \\
\hline .149 & & $\cdot 119$ & & .194 & & .199 & & $r \cdot 1$ & Irqf & سلطانى & اروميه [ץr] \\
\hline \multirow[t]{5}{*}{$\cdot / r v$} & & $\cdot / \sqrt{ } 9$ & & $\cdot 119$ & & . $/ 94$ & & $1 r 9$ & 1490 & صيادى & اروميه [سץ] \\
\hline & & & & $\cdot / \Lambda \Delta$ & & .199 & & 99 & \multirow{2}{*}{$11 \% 9$} & \multirow{2}{*}{ مهدوى زفرقندى } & \multirow{2}{*}{ مشهد [ry] } \\
\hline & & & &.$/ 99$ & &.$/ 99$ & & Ifr & & & \\
\hline & & & & & & $\cdot 19$ & 1 & IVr & Irवs & رضا يور & مشهد [ro] \\
\hline & & $\cdot 119$ & & .194 & &.$/ 91$ & & rul & Irqs & عسكرى شاهى & اصفهان [عץ] \\
\hline$\cdot 119$ & $\cdot 119$ & $\cdot 19$ & .191 & $\cdot 195$ & .190 & .199 & $\cdot 191$ & \multirow{2}{*}{ १११ } & \multirow{2}{*}{ Irag } & \multirow{2}{*}{ قليجى قوجق } & \multirow{2}{*}{ ايران [rv] } \\
\hline$\cdot / \Lambda \cdot$ & $\cdot 119$ & $\cdot / \Lambda \Delta$ & .191 & $\cdot \mid \Lambda V$ & . 194 & . 194 & .198 & & & & \\
\hline & & & $\cdot / 9$ & & .194 & &.$/ 9 F$ & AVG & Irवs & رضيى & كرمانشاه [^ץ]] \\
\hline \multirow[t]{2}{*}{$\cdot / \wedge \Delta$} & & .191 & & & & & & $r \Delta q$ & IrqV & روشنايى & همدان [qץ] \\
\hline & & & & $\cdot|\Lambda|$ & & $\cdot / A V$ & & $|\Delta V|$ & 1191 & قربانى & كرمانشاه [F.] \\
\hline
\end{tabular}


جدول r. ارزيابى كيفيت مقالات واردشده در متآناليز

\begin{tabular}{|c|c|c|c|c|c|c|c|}
\hline امتياز & نوع مطالعه & نحوة اندازهَيرى & معيارهاى ورود و & موردمطالعه & زمان انجام & محل انجام & دد مقاله \\
\hline 11 & $r$ & $r$ & 1 & $r$ & r & $r$ & 1 \\
\hline 11 & $r$ & $r$ & 1 & $r$ & r & $r$ & $r$ \\
\hline Ir & $r$ & $r$ & $r$ & $r$ & $r$ & $r$ & $r$ \\
\hline 1. & $r$ & $r$ & - & $r$ & $r$ & $r$ & r \\
\hline IT & $\Delta$ & $r$ & $r$ & $r$ & $r$ & $r$ & $\Delta$ \\
\hline 1. & r & r & - & $r$ & r & $r$ & 9 \\
\hline 1. & $r$ & $r$ & - & r & r & $r$ & v \\
\hline IT & r & $r$ & $r$ & r & r & $r$ & $\wedge$ \\
\hline 11 & $r$ & $r$ & 1 & $r$ & $r$ & $r$ & 9 \\
\hline 11 & r & r & 1 & r & r & r & 1. \\
\hline 1. & r & $r$ & - & $r$ & r & $r$ & 11 \\
\hline Ir & r & r & $r$ & r & r & $r$ & Ir \\
\hline 1. & r & $r$ & - & $r$ & $r$ & $r$ & Ir \\
\hline 11 & r & r & 1 & r & r & r & if \\
\hline 11 & $r$ & $r$ & 1 & $r$ & $r$ & $r$ & 10 \\
\hline 1. & r & r & - & r & r & $r$ & 19 \\
\hline 11 & r & r & 1 & $r$ & $r$ & $r$ & IV \\
\hline 1. & r & r & - & r & r & r & 11 \\
\hline 11 & r & r & 1 & $r$ & $r$ & $r$ & 19 \\
\hline 11 & r & $r$ & 1 & r & r & $r$ & r. \\
\hline 11 & $r$ & $r$ & 1 & $r$ & r & $r$ & $r$ \\
\hline 1. & $r$ & $r$ & - & r & r & $r$ & Tr \\
\hline 1. & r & $r$ & - & $r$ & $r$ & $r$ & r \\
\hline 1. & r & r & - & r & r & r & TF \\
\hline 1. & $r$ & $r$ & - & $r$ & $r$ & $r$ & $r \Delta$ \\
\hline Ir & r & $r$ & $r$ & r & $r$ & $r$ & rq \\
\hline$\wedge$ & - & r & - & $r$ & $r$ & $r$ & TV \\
\hline 1. & r & $r$ & - & r & r & $r$ & rA \\
\hline 1. & $r$ & $r$ & - & $r$ & $r$ & $r$ & $r q$ \\
\hline$\wedge$ & - & r & - & r & $r$ & $r$ & $r$. \\
\hline$\wedge$ & - & $r$ & - & $r$ & $r$ & $r$ & $\mu_{1}$ \\
\hline 1. & & $r$ & - & r & r & r & rT \\
\hline 1. & $r$ & $r$ & - & $r$ & $r$ & $r$ & r \\
\hline
\end{tabular}




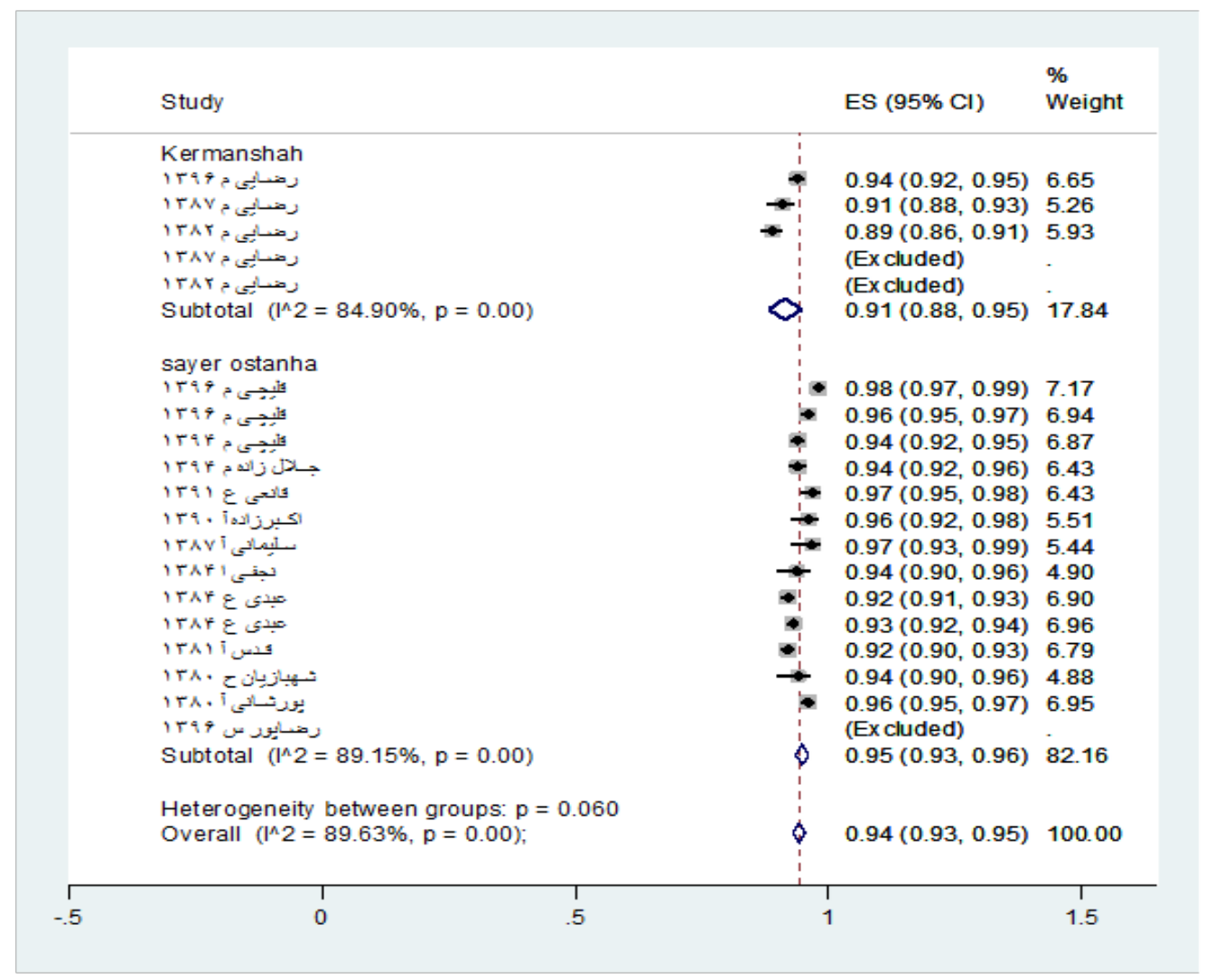

شكل r. بر آورد ميزان بقاى يكساله بيماران پِيوند كليه در مطالعات موردبررسى به تفكيك استان كرمانشاه با ساير استانهاى كشور (هركدام ازيارهخطها

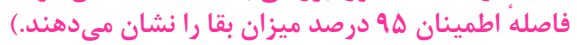

نمودارهاى مربوط به ميزان بقاى سه، رِنج و ده سال يِيوند كليه در ييوست ارائه شده است.

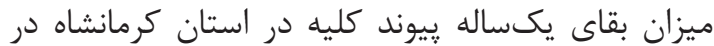

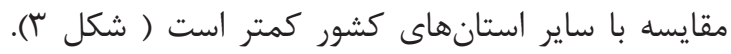

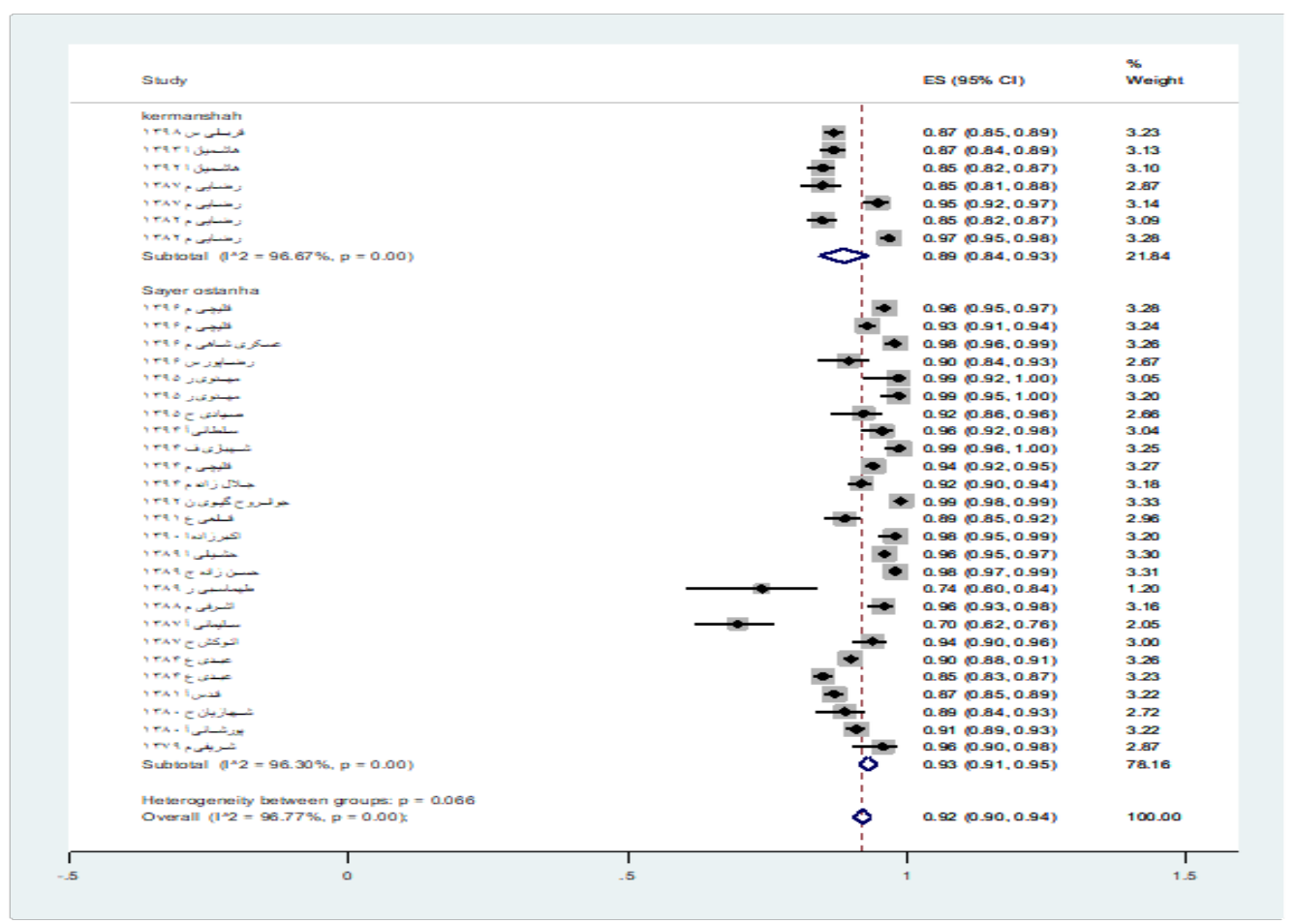

شكل ". بر آورد ميزان بقاى يك ساله كليه يِوندى در مطالعات مورد بررسى به تفكيك استان كرمانشاه با ساير استانهاى كشور (هركدام ازيارهخطها فاصله اطمينان هو درصد ميزان بقا را نشان مى دهنيك اسندان

q 
ينجساله ميزان بقاى كليئ يِيوندى در استان كرمانشاه بيشتر

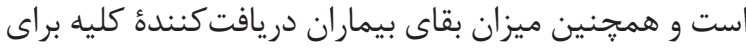

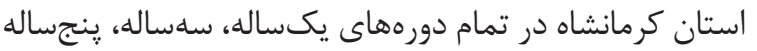

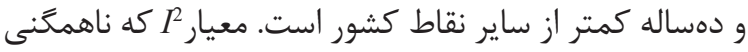

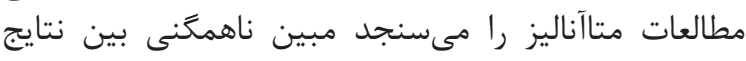
مطالعات است (بيش از له VD: ناهمكنى زياد).

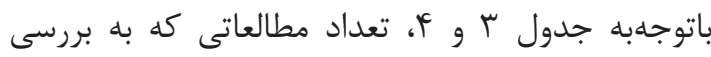

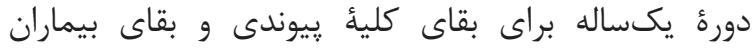

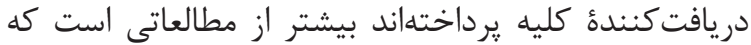

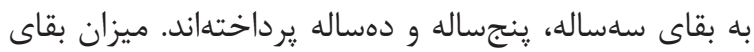

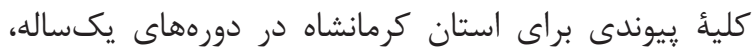

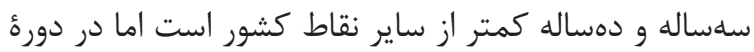

جدول ب. ميزان بقاى بيماران دريافتكنندهُ بِيوند كليه بهدست آمده از مطالعات به تفكيك استان كرمانشاه و ساير نقاط

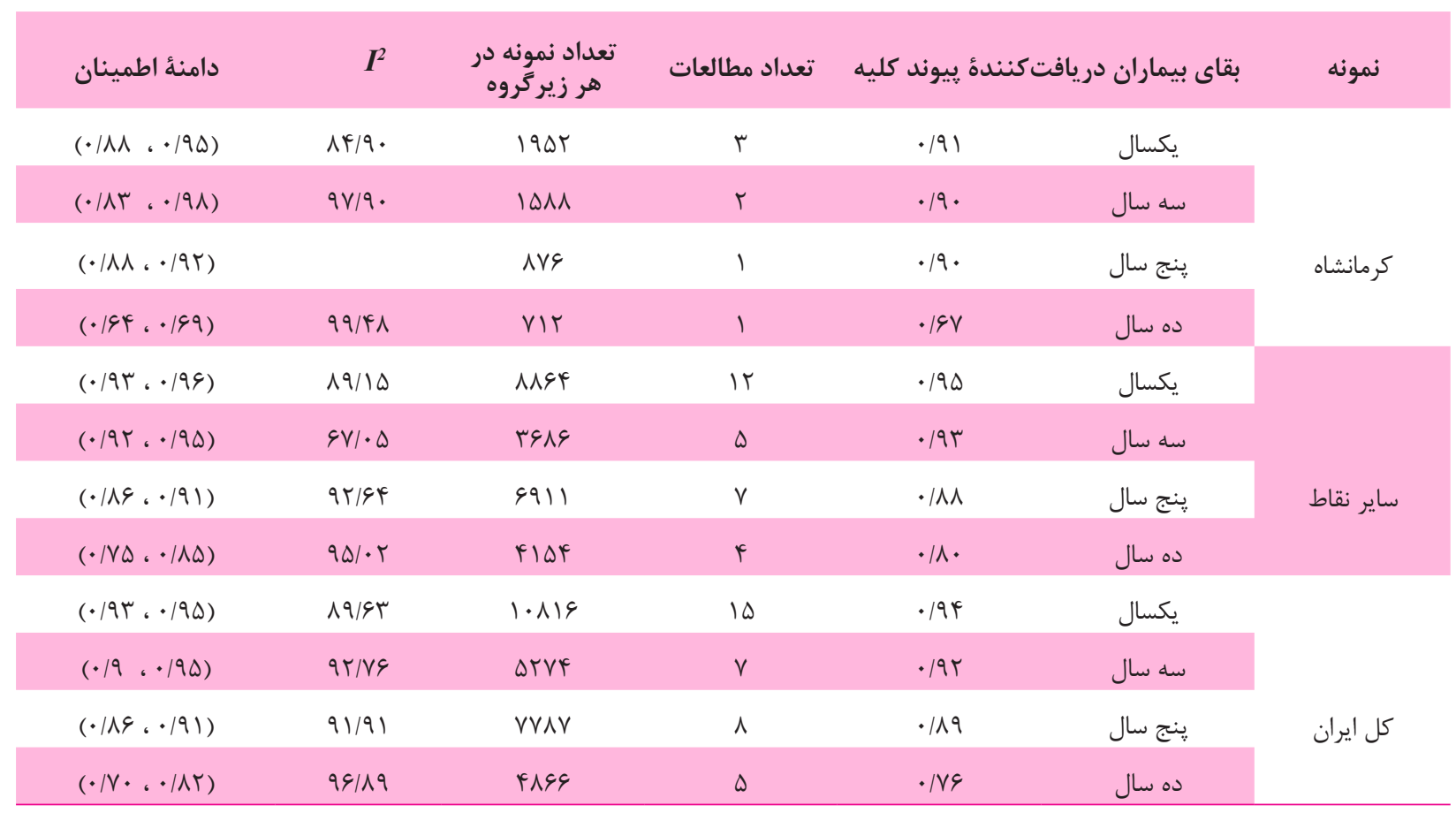

جدول F. ميزان بقاى كليئ بييوندشده بهدستآمده از مطالعات به تفكيك استان كرمانشاه و ساير نقاط

\begin{tabular}{|c|c|c|c|c|c|c|}
\hline دامنهُ اطمينان & $I^{2}$ & تعر زير نمونه دره & تعداد مطالعات & \multicolumn{2}{|c|}{ بقاى بيماران دريافت كننده هِيوند } & 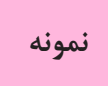 \\
\hline$(\cdot / \Lambda F, \cdot / 9 r)$ & $94 / 9 V$ & 4109 & $\Delta$ & $\cdot 119$ & يك سال & \multirow{4}{*}{ كرمانشاه } \\
\hline$(\cdot / \vee \Delta, \cdot / \Lambda \vee)$ & $9 \& / \Delta \Lambda$ & rVqQ & f & $\cdot|\Lambda|$ & سه سال & \\
\hline$(\cdot / \vee /, \cdot / V \varphi)$ & १९/१५ & IDIT & r & $\cdot / V F$ & ينج سال & \\
\hline$(\cdot / F \mu, \cdot / F \Lambda)$ & १९/१९ & VIr & 1 & $\cdot / 49$ & ده سال & \\
\hline$(.191, . .190)$ & $q \Leftrightarrow / \pi$. & $|r \Delta \Lambda|$ & rI &.$/ 94$ & يك سال & \multirow{4}{*}{ ساير نقاط } \\
\hline$(\cdot / 4 V . \cdot / 9 r)$ & $9 \% / \cdot \Lambda$ & VETY & If & .19 & سه سال & \\
\hline$(\cdot / \vee 9, \cdot / \wedge \varepsilon)$ & $9 \Lambda / \Upsilon \wedge$ & 1111 & 11 & . INT & ينج سال & \\
\hline$(\cdot / 9 Y, \cdot / V \Lambda)$ & $91 / 91$ & NTrT & 1. & $\cdot / \mathrm{V}$ & ده سال & \\
\hline$(\cdot / 9 \cdot, . / 94)$ & $q \& / V V$ & IVVE. & re &.$/ 95$ & يك سال & \multirow{4}{*}{ كل ايران } \\
\hline$(\cdot / \wedge \Delta, \cdot / 9 \cdot)$ & Q४/QT & IITIV & 11 & $\cdot / \Lambda V$ & سه سال & \\
\hline$(\cdot / \vee \wedge, \cdot / \wedge \Delta)$ & $q \Lambda / T V$ & TMTK & $r \cdot$ & . MT & ينج سال & \\
\hline$(\cdot / \Delta V, \cdot / V \varepsilon)$ & ११/५। & NqFF & 11 & $\cdot 19 \mathrm{~V}$ & ده سال & \\
\hline
\end{tabular}


در استان كرمانشاه VF درصد است و ميزان بقاى ينجساله

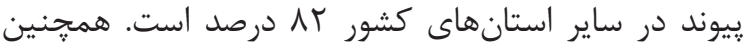

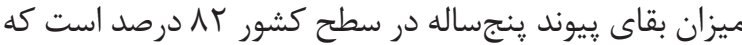

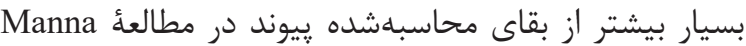

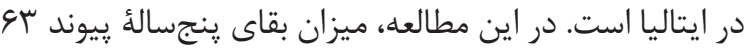

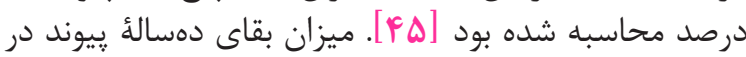

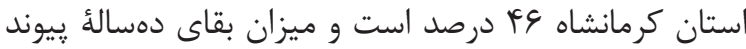

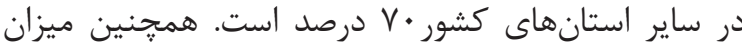

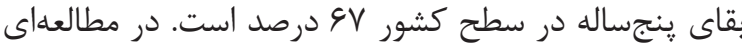

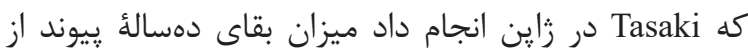

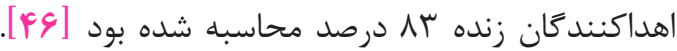

باتوجلبه نتايج مذكور بقاى يكساله، سهساله و دهسالة

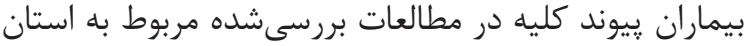

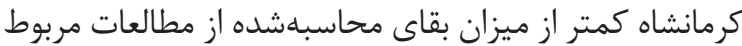

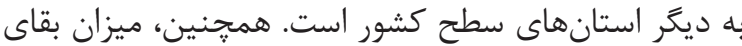

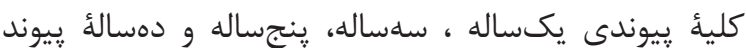

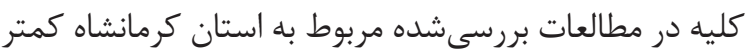

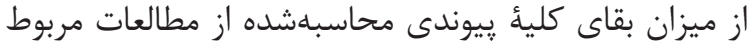

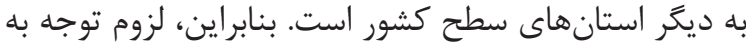

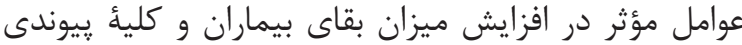

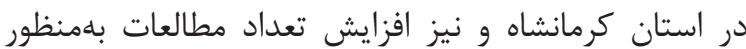

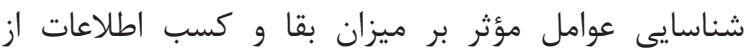
كاستى هاى موجود، ضرورى به نظر مئر ميرسد.

$$
\text { سياسخَزارى موجن }
$$

از معاونت يزوهشى دانشكده بهداشت و معاونت تحقيقات و فناورى دانشعاه جهت حمايت از انجام اين مطالعه تشكيت تشكر و تصفيقات

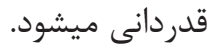

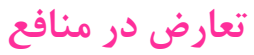
بين نويسندكان هيج كَونه تعارضى در منافع وجود ندارد.

\section{References}

1. Ziemba JB, Matlaga BR. Epidemiology and economics of nephrolithiasis. Investig Clin Urol. 2017; 58:299-306. [DOI:10.4111/ icu.2017.58.5.299] [PMID] [PMCID]

2. Parmar MS. Kidney stone. BMJ. 2004; 328 (7453): 1420-4. [DOI:10.1136/ bmj.328.7453.1420] [PMID] [PMCID]

3. Stoller ML. Urinary stone disease. In: Tanagho EA, McAninch JW, editors. Smith's General Urology. 16th ed. New York: McGraw Hill; 2004. pp. 256-86.

4. K N Stamatiou 1, V I Karanasiou, R E La-
نتيجه كيرى و بحث

براساس نتايج بهدستآمده در اين تحقيق ميزان بقاى يكساله بيمار در استان كرمانشاه

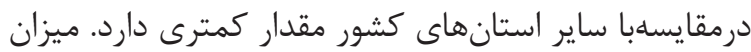

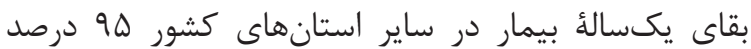

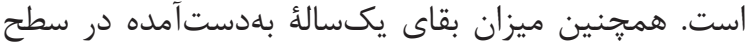

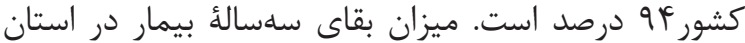

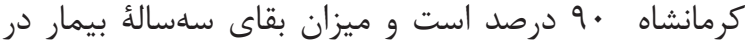

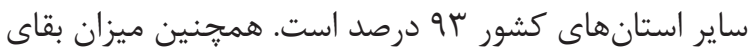

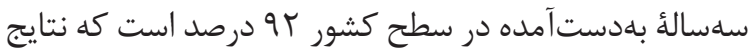

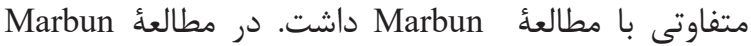

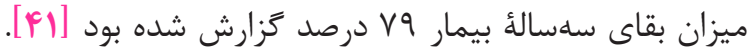

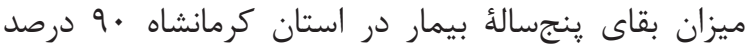

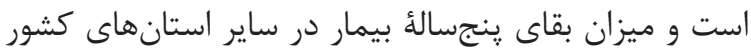

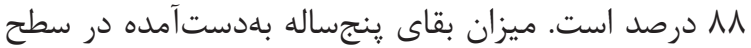

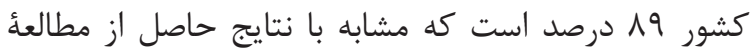

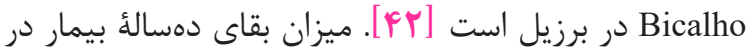

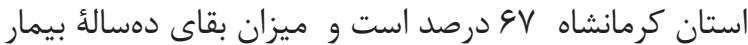

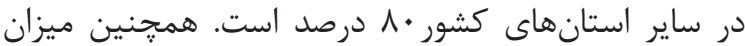

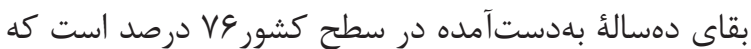

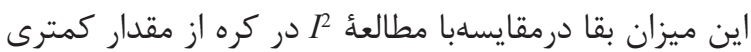

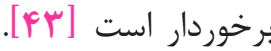

نتايج حاصل از اين تحقيق نشان داد ميزان بقاى ييوند

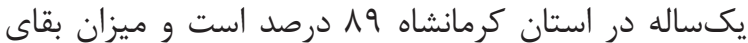

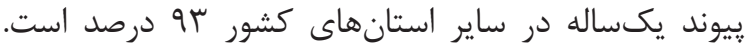

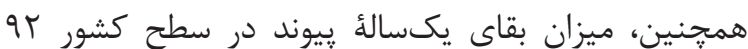

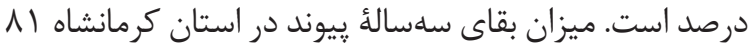

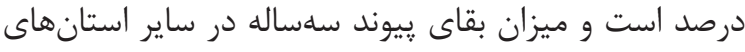

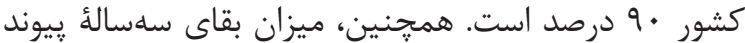

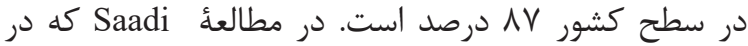

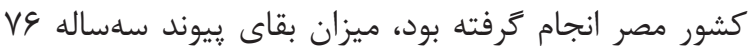

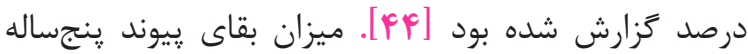

croix, N G Kavouras, V T Papadimitriou, C Chlopsios, F A Lebren, F Sofras

5. Prevalence of urolithiasis in rural Thebes, Greece. Rural and Remote Health. 2006; 6:610.

6. Fan X, Kalim S, Ye W, Zhao S, Ma J, Nigwekar SU, Chan KE, et al. Urinary stone disease and cardiovascular disease risk in a rural Chinese population. Kidny Int Rep. 2017; 2 (6):1042-9. [DOI:10.1016/j. ekir.2017.06.001] [PMID] [PMCID]

7. Lotan $\mathrm{Y}$, Daudon $\mathrm{M}$, Bruyère $\mathrm{F}$, Talaska G, Strippoli G Johnson RJ, et al. Impact of fluid intake in the prevention of uri- 
nary system diseases: a brief review. Current Opinion in Nephrology and Hypertension. 2013; 22:S1-S10. [DOI:10.1097/ MNH.0b013e328360a268] [PMID]

8. Scales CD, Smith AC, Hanley JM, Saigal CS. Prevalence of kidney stones in the united states. Eur Uro. 2012; 62:1605. [DOI:10.1016/j.eururo.2012.03.052] [PMID] [PMCID]

9. Lineman JE, Smith LH, Woods JR, Newman DM. Urinary Calculi: ESWL endourology and medical therapy. In: Lingeman JE, Smith LH, Woods JR, Newman DM. Ureteral Calculi. 3nd ed. Philadelphia: Lea and Febiger, 1989; 192-205.

10. Griffin DG. A review of the heritability of idiopathic nephrolithiasis. J Clin Pathol. 2004; (57):793-6. [DOI:10.1136/ jcp.2003.014886] [PMID]

11. Basiri A, Shakhssalim N, Khoshdel A, Pakmanesh H, Radfar M. Drinking water composition and incidence of urinary calculus introducing a new index. IJKD. 2011; 5:15-20.

12. Trinchieri A. Epidemiology of urolithiasis: an update. Clin Cases Miner Bone Metab. 2008; 5:101-6.

13. Pearle MC, Partin AW, Peter CA. Urinary Lithiasis. In: Wein AJ, Kavoussi LR, Novick AC. Campbell Walsh Urology. 3rd edition. Saunders. 2007; 2: 1363-1525.

14. Saxena A, Sharma R K. Nutritional aspect of nephrolithiasis. Indian J Urol [serial online] 2010 [cited 2020 Jun 14];26:523-30. [DOI:10.4103/0970-1591.74451] [PMID]

15. Siener R, Jahnen A, Hesse A. Influence of a mineral water rich in calcium, magnesium and bicarbonate on urine composition and the risk of calcium oxalate crystallization. Eur J Clin Nutr. 2004; 58(2):270-6. [DOI:10.1038/sj.ejcn.1601778] [PMID]

16. Borghi L, Meschi T, Amato F, Briganti A, Novarini A, Giannini A. Urinary volume, water and recurrences in idiopathic calcium nephrolithiasis: a 5-year randomized prospective study. J Urol. 1996; 155:839-43. [DOI:10.1016/S0022-5347(01)66321-3]

17. Curhan GC, Willet WC, Rimm EB,
Stampfer MJ. A prospective Study Of dietary calcium and other nutrients and the risk of symptoms kidney stones. N Eng J Med. 1993; 328(12):833-8. [DOI:10.1056/ NEJM199303253281203] [PMID]

18. Blaser MJ, Falkow S. What are the consequences of the disappearing human microbiota? Nat Rev Microbiol. 2009; 7:887. [DOI:10.1038/nrmicro2245] [PMID]

19. Poore CA, Coker C, Dattelbaum JD, Mobely HL. Identification of the domains of UreR, an araC-like transcriptional regulator of the urease gene cluster in Proteus mirabilis. J Bacteriol. 2001; 183(15):4526-35. [DOI:10.1128/JB.183.15.4526-4535.2001] [PMID] [PMCID]

20. Watzl B. Anti-inflammatory effects of plant-based foods and of their constituents. Int J vitam Nutr Res. 2008; 78:2938. [DOI:10.1024/0300-9831.78.6.293] [PMID]

21. IBM SPSS Statistics software help.

22. Shirazi F, Shahpourian F, Houshiarrad A, Hosseini F, Khachian A, Heidari S. Association between dietary factors and renal stones in adults. Iranian Journal of Nutrition Sciences \& Food Techn. 2009; 4:5765.

23. Johri N, Cooper B, Robertson W, Choong S, Rickards D, Unwin R. An Update and practical Guide to Renal Stone Management. Neph Clin Prac. 2010; 116:c159-c71. [DOI: 10.1159/000317196] [PMID]

24. Romero VH, Assimos D. Kidney Stones: A Global Picture of Prevalence, Incidence, and Associated Risk Factors. 2010; 12(2-3):e86-e96.

25. Casper D. Principles of Internal Medicine, Kidney Diseases and Urinary Tract Diseases. Translated by Ida Parafi, 19th Edition. 2015; Publisher NOOR DANASH

26. Asplin JR. Obesity and Urolithiasis. Ad Chr Kid Dis. 2009; 16:11-20. [DOI:10.1053/j. ackd.2008.10.003] [PMID]

27. Heilberg IP, Goldfarb DS. Optimum Nutrition for Kidney Stone Disease. Adv Chron Kid Dis. 2013; 20:165-74. [DOI:10.1053/j. 


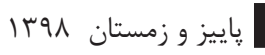

فصلنامه علمى يروهشى تحقيقات در ارولوزى لدوره ب شماره r

ackd.2012.12.001] [PMID]

28. Lewandowski S, Rodgers AL. Idiopathic calcium oxalate urolithiasis: risk factors and conservative treatment. Clinica Chimica Acta. 2004; 345:17-34. [DOI:10.1016/j. cccn.2004.03.009] [PMID]

29. Sellaturay S, Fry C. The metabolic basis for urolithiasis Surgery. Iranian Journal of Epidemiology 2008; 26:136-40. [DOI:10.1016/j.mpsur.2008.03.002]

30. Latan Y, Daudon M, Bruyere F, Talaska G, Strippoli G, Johnson RJ, et al. Impact of fluid intake in the prevention of urinary system diseases: a brief review. Curt Op Nephrol Hyper. 2013; 22:51. [DOI:10.1097/ MNH.0b013e328360a268] [PMID]

31. Grases F, Saez-Torres C, Rodriguez A. Urinary phytate (Myo-inositol hexaphosphate) in healthy school children and risk of nephrolithiasis. J Ren Nutr. 2014; 24:219-23. [DOI:10.1053/j.jrn.2014.03.004] [PMID]

32. Barnela S, Soni S, Saboo S, Bhansali A. Medical management of renal stone. Ind J Endocr Met. 2012; 16:236-9. [DOI:10.4103/2230-8210.93741] [PMID] [PMCID]

33. Ahmadzadeh A, Jamshidi Moghaddam Z. Prevalence, Type and Symptoms of Urinary Stones in Children Under 15 Years with Symptomatic Urinary Tract Infection. Scentific J Med. 2005; 4(2):155-162.

34. Kohanzad S. Urology at a glance. Tehran: NASHR DANASH; 1994.

35. Bokharposh M, Heidari A. Study of dietary intake of fluids in patients with urinary stones referring to Razi Hospital in Rasht. Hamadan Fac Nur Mid. 20(11):15-20.

36. Taghdisi MH, Nejadsadeghi E. The Effect of Health Education Based on Health Belief Model on Behavioral Promotion of Urinary Infection Prevention in Pregnant Women. J Commun Health Res Cent. 2012; 2:126-36. 